\title{
The lin-4 Gene Controls Fat Accumulation and Longevity in Caenorhabditis elegans
}

Chun Zhu ${ }^{1, \dagger}$, Chen-Bo Ji ${ }^{2, \dagger}$, Chun-Mei Zhang ${ }^{1}$, Chun-Lin Gao ${ }^{2}$, Jin-Gai Zhu ${ }^{2}$, Da-Ni Qin ${ }^{2}$, Chun-Zhao Kou ${ }^{2}$, Guan-Zhong Zhu ${ }^{2}$, Chun-Mei Shi ${ }^{2}$ and Xi-Rong Guo ${ }^{1, *}$

1 Department of Pediatrics, Nanjing Maternal and Child Health Hospital of Nanjing Medical University, No.123 Tianfei Road, 210004 Nanjing, China;

E-Mails: zhifangxibao@163.com(C.Z.); zhangcm79@163.com (C.-M.Z.)

2 Institute of Pediatrics, Nanjing Medical University, No.140 Hanzhong Road, 210029 Nanjing, China; E-Mails: Jiliu1036@163.com (C.-B.J.); shuangmu34@ sina.com (C.-L.G.); zhujingai1983@163.com (J.-G.Z.); qindani@yahoo.com.cn (D.-N.Q.);

kou19850112@163.com (C.-Z.K); aerozgz@yahoo.com.cn (G.-Z.Z.); shichunmei_87@163.com (C.-M.S.)

$\dagger$ These authors contributed equally to this work.

* Author to whom correspondence should be addressed; E-Mail: xrguo@njmu.edu.cn; Tel.: +86-25-868-629-96; Fax: +86-25-868-629-96.

Received: 10 October 2010; in revised form: 5 November 2010 / Accepted: 18 November 2010 / Published: 25 November 2010

\begin{abstract}
Previous studies have determined that lin-4, which was the first miRNA to be discovered, controls the timing of cell fate determination and life span in Caenorhabditis elegans. However, the mechanism of lin-4 involvement in these processes remains poorly understood. Fat storage is an essential aspect of the life cycle of organisms, and the function of lin-4 in fat accumulation is not clear. In this study, we showed that the fat content is reduced remarkably in C. elegans lin-4 mutants. Quantitative RT-PCR analysis revealed a considerable decrease in the levels of SBP-1 and OGA-1 mRNA in lin-4 mutants. We also showed that lin-4 mutants have a significantly shorter life span than wild-type worms. DCF assay experiments showed that the reactive oxygen species (ROS) levels increased and mitochondrial DNA (mtDNA) copy number decreased in loss-of-function lin-4 mutants. These mutants also showed attenuation of locomotion. Taken together, our findings suggest that lin-4 may play an important role in regulating fat accumulation and locomotion and that lin-4 may control the life span of $C$. elegans by mediating ROS production.
\end{abstract}


Keywords: lin-4; Caenorhabditis elegans; fat accumulation; life span; locomotion

\section{Introduction}

MicroRNAs (miRNAs) are 18-25-nucleotide-long single-stranded RNAs that are involved in the post-transcriptional regulation of gene expression by base pairing with complementary sequences in the 3' untranslated regions (3' UTRs) of protein-coding transcripts. This interaction leads to the inhibition of translation [1,2]. Although the biological functions of most miRNAs are unknown, miRNAs are predicted to regulate up to $30 \%$ of the genes within the human genome [3]. Emerging evidence also suggests that miRNAs play important regulatory roles in a variety of biological processes, including adipocyte differentiation, metabolic integration, insulin resistance, and appetite regulation [4]. Although the Caenorhabditis elegans genome encodes over 100 miRNAs, the functions of only five have been elucidated, and less than 15 valid $C$. elegans target genes are known [5]. As the first miRNA discovered, lin-4 acts as a developmental switch that controls the timing of cell fate determination during larval transitions in C. elegans and regulates the life span of the adults [6,7]. However, our current knowledge of the connection between lin-4, life span, and locomotion in C. elegans is very limited.

The increasing prevalence of obesity has significant implications for the health of humans because obesity is associated with serious co-morbidities, including type 2 diabetes mellitus, hyperlipidemia, and hypertension [8]. Obesity results from a prolonged imbalance in energy homeostasis between caloric intake and expenditure. Animal models have provided an understanding of the basic parameters that regulate the components of energy balance [9]. The use of invertebrates such as C. elegans, which is an excellent model for many biological processes, may be a more efficient method of screening research materials and a powerful system for analyzing the mechanisms of fat storage. Moreover, many components regulating human metabolism, including the biochemical pathways of fats, carbohydrates, and protein synthesis, are conserved in the nematode C. elegans $[10,11]$. However, the relationship between lin-4 and fat accumulation is unknown.

In this study, we compared fat accumulation, reactive oxygen species (ROS) levels, mitochondrial DNA (mtDNA) copy number, life span, and locomotion in lin-4 mutants with those in wild-type C. elegans. We also tested whether feeding glucose and fatty acids affects fat storage in C. elegans. In addition, we used the real-time quantitative reverse transcriptase-polymerase chain reaction (RT-PCR) to determine the mRNA expression of SBP-1 and OGA-1, which are key genes in regulating and controlling the amount and composition of fat $[12,13]$.

\section{Results and Discussion}

\subsection{The Fat Content Is Remarkably Reduced in lin-4 Mutants}

As shown in Figure 1, when worms were fed a normal diet of E. coli OP50 bacteria, animals with a loss-of-function mutation in lin-4 showed fat content that was significantly lower than that in wild-type animals. After adding glucose and fatty acid to culture dishes containing C. elegans, we found an 
increase in fluorescence in Nile red staining, thereby indicating an increase in intracellular fat. However, fat levels were also reduced considerably in lin-4 mutants compared to wild-type animals. To determine whether the key genes in the metabolic pathways for fatty acid synthesis in nematodes are involved in regulating fat accumulation, we measured mRNA levels of SBP-1 and OGA-1 by using quantitative RT-PCR analysis. We found that both SBP-1 and OGA-1 mRNA levels were reduced in lin-4 mutants, especially SBP-1(Figure 2).

Figure 1. lin-4 regulates fat storage. (A) Nile red staining of wild-type (N2) and lin-4 mutant (e912) worms fed a normal diet of E. coli OP50 bacteria (left panel) or OP50 bacteria supplemented with glucose (middle) or fatty acid (right). Scale bar: $50 \mu \mathrm{m} . n=40$ ( $n$ : number); (B) Quantification of the Nile red staining of fat in wild-type (N2) and lin-4 mutant (e912) worms. $n=40$. Error bars indicate standard error. ${ }^{*} p<0.05$.

(A)

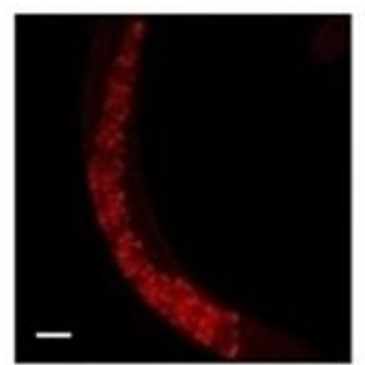

wild-type (N2)

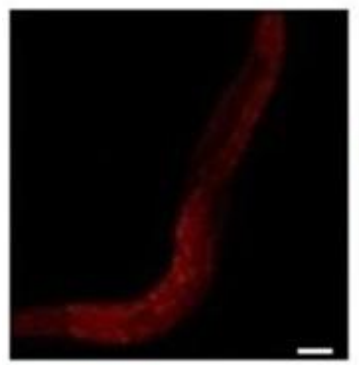

$\operatorname{lin}-4(\mathrm{e} 912)$

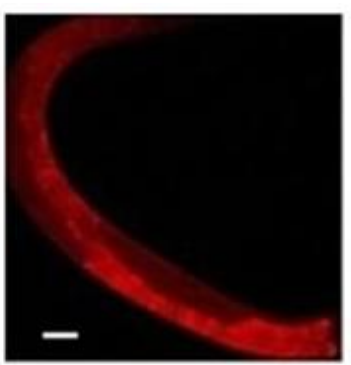

Wild-type (N2) + Glucose

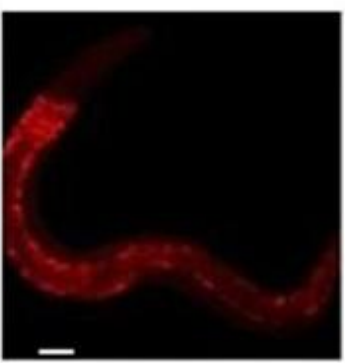

$\operatorname{lin}-4(e 912)+$ Glucose

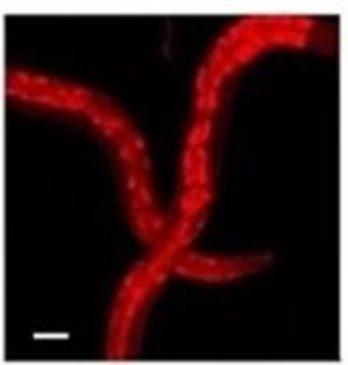

Wild-type (N2) + Fatty acid

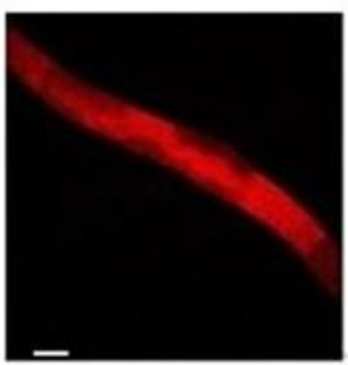

lin-4(e912) + Fatty acid

(B)

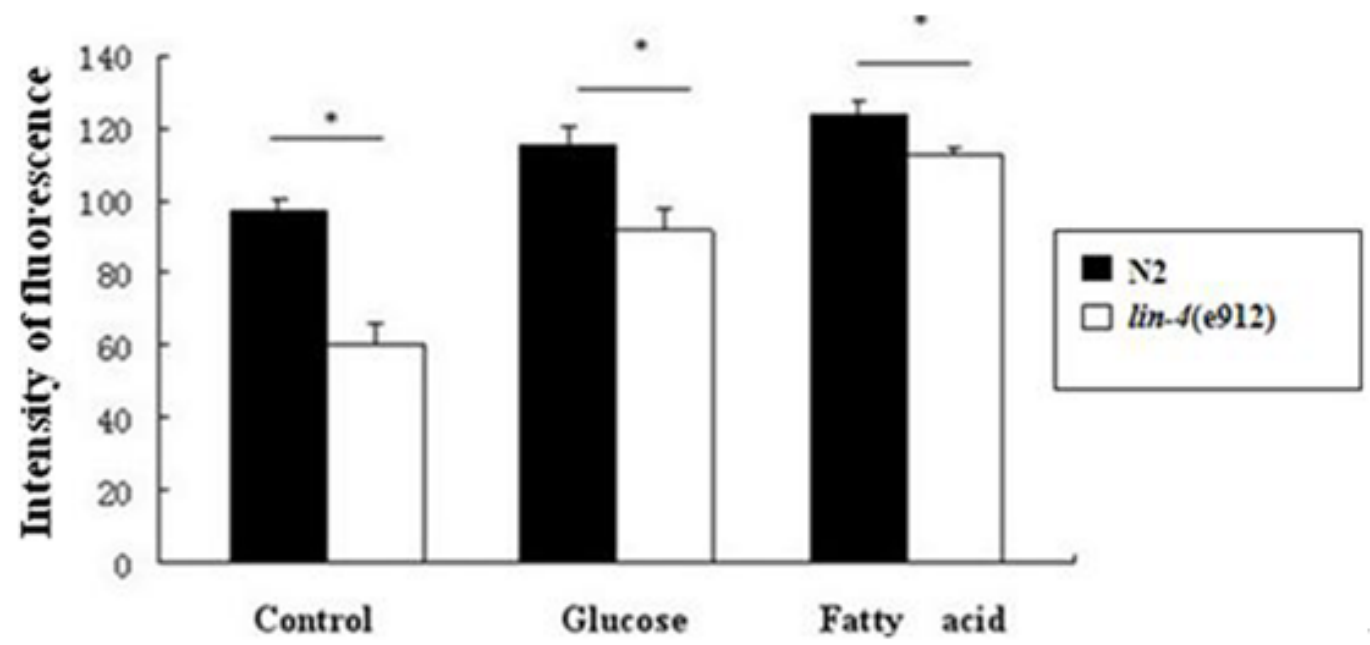


Figure 2. The expression of central regulation factors involved in fat storage control in nematodes. (A) Quantitative real-time PCR of sbp-1 in wild-type (N2) and lin-4 mutant (e912) worms. $n=50$ ( $n$ : number); (B) Quantitative real-time PCR of oga-1 in wild-type (N2) and lin-4 mutant (e912) worms. $n=40$. Error bars indicate standard error. ${ }^{*} p<0.05$.
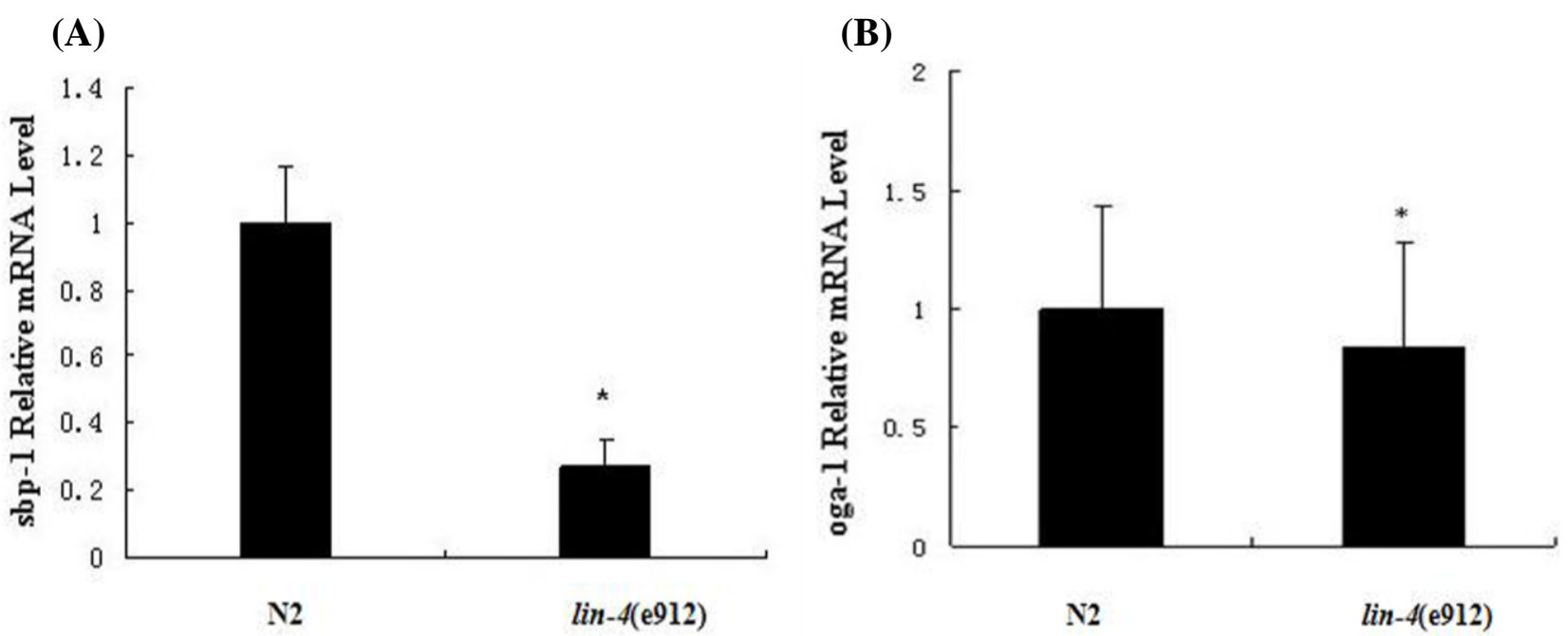

\subsection{ROS Accumulation and Decreased Copy Number of mtDNA in lin-4 Mutants}

DCF assay experiments revealed that worms with a loss-of-function mutation in lin-4 showed ROS accumulation (Figure 3). We examined the mtDNA copy number in lin-4 mutants and wild-type animals by performing real-time PCR. The mtDNA copy number in lin-4 mutants was significantly lower than that in the wild-type control (Figure 4).

Figure 3. The effect of lin-4 on ROS level. (A) Intracellular ROS in C. elegans were measured using 2,7-dichlorofluorescein diacetate (DCF-DA; Molecular Probes). Sixty animals from each group were analyzed $(n=60)$. Scale bar: $100 \mu \mathrm{m} ;(\mathbf{B})$ results are expressed as mean $\pm \mathrm{SD}$ of relative fluorescence units (RFU). ${ }^{*} p<0.05$.

(A)

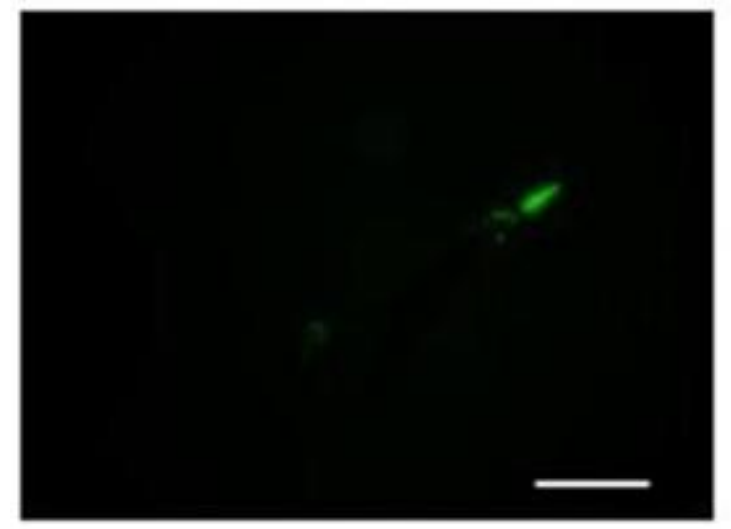

N2

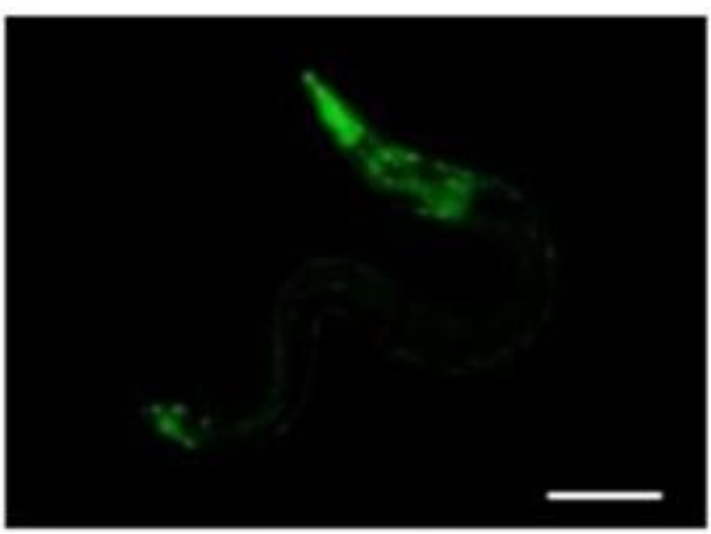

lin-4(e912) 
Figure 3. Cont.

(B)

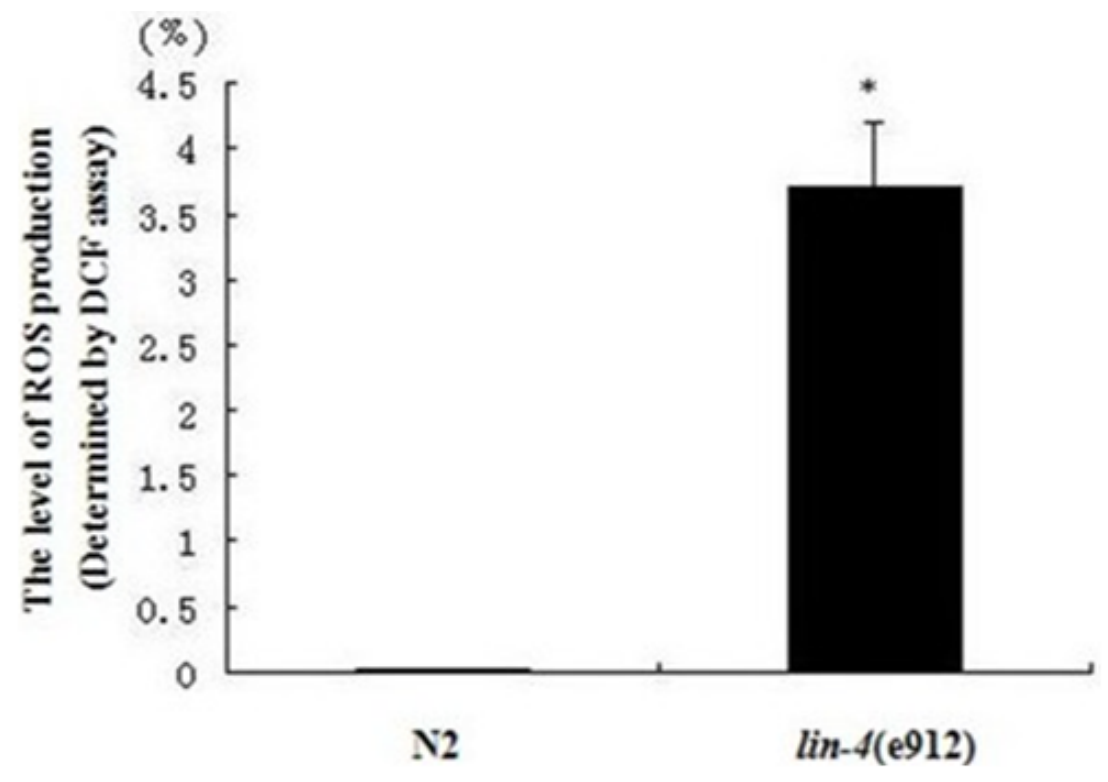

Figure 4. Effects of lin-4 mutation on copy number of mtDNA. The mtDNA copy number was measured in wild-type (N2) and lin-4 mutant (e912) worms. Values represent the mtDNA copy number per worm. At least three replicates were performed. ${ }^{*} p<0.05$.

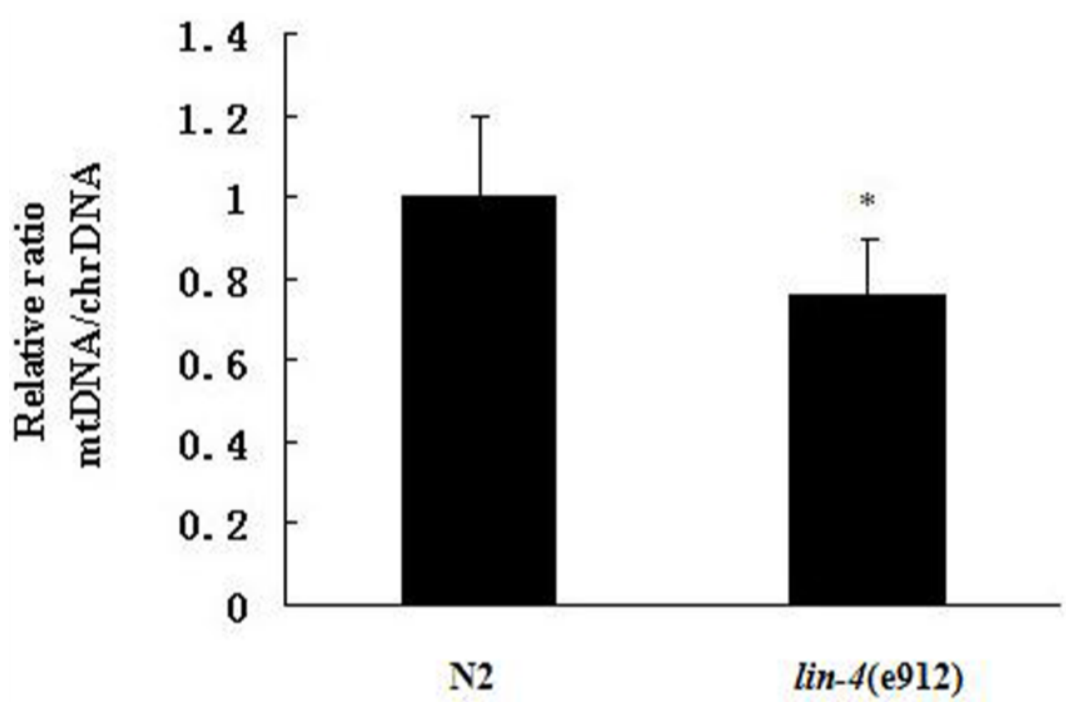

2.3. Effect of lin-4 on the Life Span and Locomotion of C. elegans

As seen in Figure 5A, the mean and maximum life span of wild-type worms grown on NGM plates containing paraquat were, respectively, $7.64 \mathrm{~d}(p<0.01)$ and $10 \mathrm{~d}(p<0.01)$ lower than the corresponding values for wild-type worms grown on NGM plates. The mean and maximum life span of lin-4 mutants grown on NGM plates containing paraquat were, respectively, $4.77 \mathrm{~d}(p<0.01)$ and $8 \mathrm{~d}(p<0.01)$ lower than the corresponding values for lin-4 mutants grown on NGM plates. The mean and maximum life span of worms with a loss-of-function mutation in lin-4 were, respectively, $7.29 \mathrm{~d}(p<0.01)$ and $9 \mathrm{~d}(p<0.01)$ lower than the corresponding values for the wild-type worms. 
Further, the mean and maximum life span of lin-4 mutants grown on NGM plates containing paraquat were, respectively, $4.42 \mathrm{~d}(p<0.01)$ and $7 \mathrm{~d}(p<0.01)$ lower than the corresponding values for the wild-type worms grown on NGM plates containing paraquat. The mean and maximum life span of wild-type worms grown on NGM plates containing paraquat were, respectively, $0.35 \mathrm{~d}(p>0.05)$ and $1 \mathrm{~d}(p>0.05)$ lower than the corresponding values for lin-4 mutants grown on NGM plates. The mean and maximum life span of lin-4 mutants grown on NGM plates containing paraquat were, respectively, $12.06 \mathrm{~d}(p<0.01)$ and $17 \mathrm{~d}(p<0.01)$ lower than the corresponding values for wild-type worms grown on NGM plates. Additionally, assessment of the body-bend behavior of $C$. elegans showed that a loss-of-function mutation in lin-4 caused severe movement defects (Figure 5B).

Figure 5. Effects of lin-4 mutation on life span and locomotion. (A) Red, survival of wild-type (N2); blue, survival after addition of paraquat to culture dishes of N2; green, survival of lin-4 mutants (e912); purple, survival after addition of paraquat to culture dishes of lin-4 mutants (e912). N2: $n=95, m=16.71, M=25$. lin-4 (e912): $n=100, m=9.42$, $M=16$. N2 + Paraquat: $n=115, m=9.07, M=15$. lin-4 (e912) + Paraquat: $n=112$, $m=4.65, M=8$. ( $n$ : number; $m$ : mean life; $M$ : maximum life); (B) N2 and lin-4 (e912) showed a mean of 85 and 58 body bends, respectively, over 1 minute. ${ }^{*} p<0.05$.

(A)

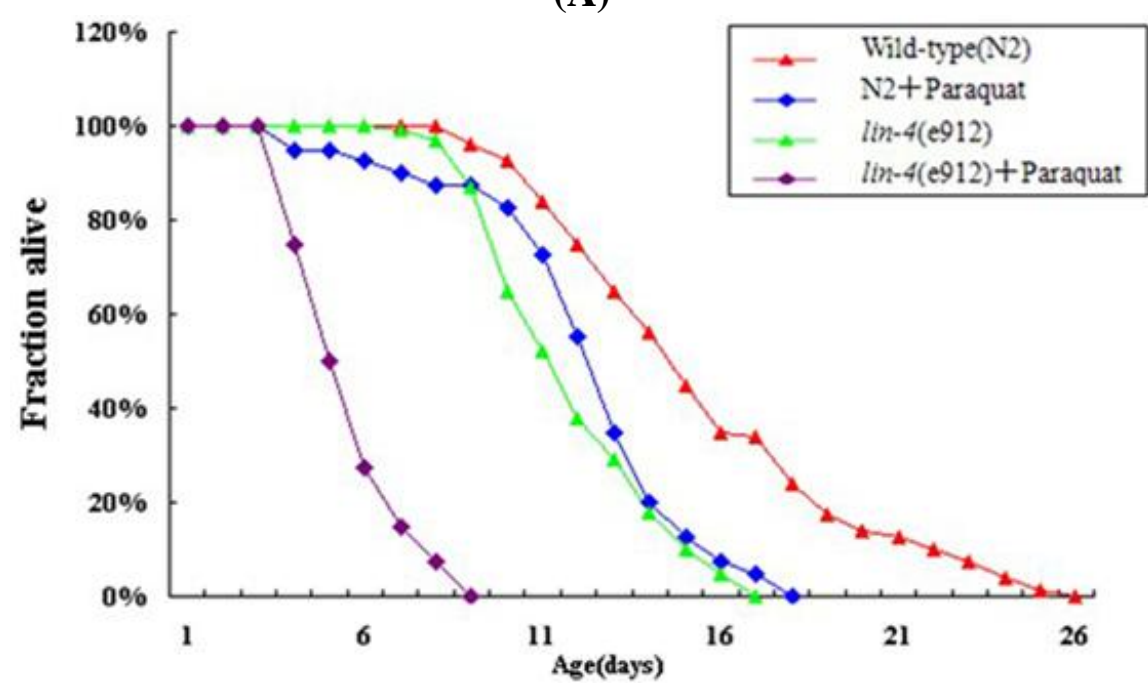

(B)

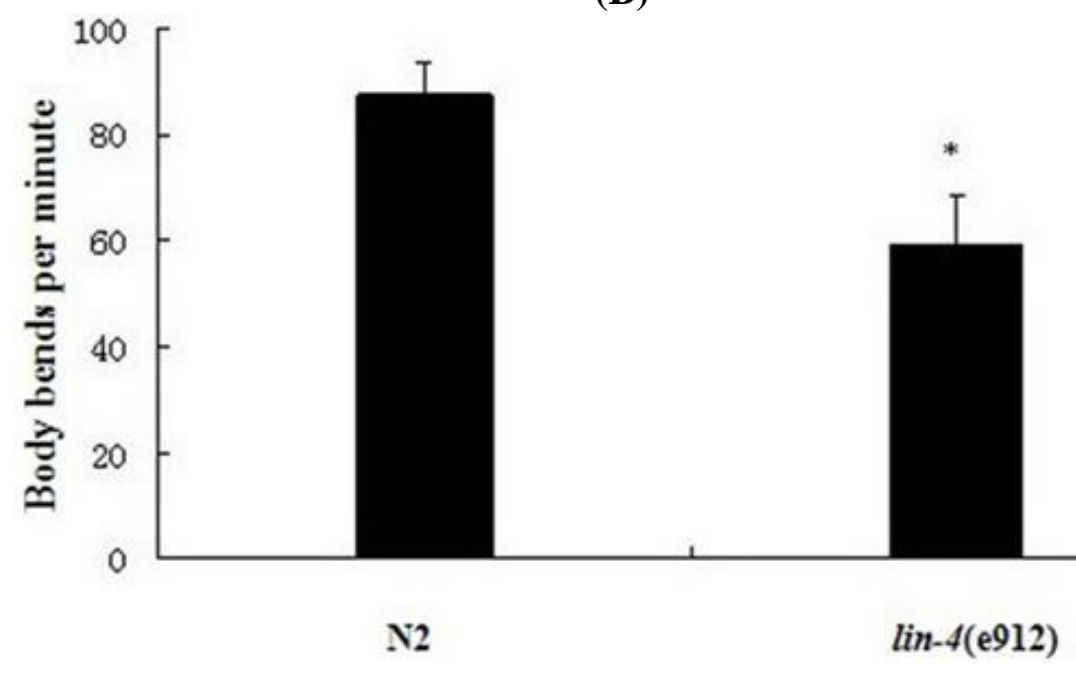




\subsection{Discussion}

In the present study, we showed that lin-4 mutants had a remarkably reduced fat content and that this phenomenon also occurred when glucose or fatty acid was used as an energy source. SBP-1 is a homolog of the mammalian transcription factor Sterol response element binding protein (SREBP). SREBP is a key transcriptional regulator in the fat and sterol synthesis pathways [14]. Previous studies have shown that loss-of-function mutations in $C$. elegans SREBP are possibly correlated with decreased fat storage $[10,15]$. $O$-linked $N$-acetylglucosamine $(O$-GlcNAc) is thought of as a dynamic nuclear and cytosolic modulator of transcriptional and signal transduction events [16]. OGA is a key enzyme regulating $O$-GlcNAc cycling and is highly conserved in eukaryotic evolution from Drosophila melanogaster and C. elegans to rodents and man [13]. A recent study suggested that active cycling of $O$-GlcNAc by OGA-1 was required to maintain normal fat reserves in worms. In contrast, reduced fat accumulation was seen in mutant strains [13]. We speculate that the remarkable reduction in fat accumulation in lin-4 mutants is due to the reduction in SBP-1 and OGA-1 mRNA levels.

Many studies have addressed the effect of lin-4 on the life span of $C$. elegans, while none has reported the relationship between ROS and lin-4. We assayed lin-4 mutants and wild-type worms for intracellular ROS and mtDNA copy number and found that mutations in lin-4 resulted in ROS accumulation and a decreased mtDNA copy number. Worms with a loss-of-function mutation in lin-4 had a life span that was significantly shorter than that of wild-type worms. Paraquat is a bipyridyl herbicide that consumes oxygen and generates superoxides [17]. We added paraquat to culture plates and observed a considerable shortening in life span, especially in lin-4 mutants. This result shows that lin-4 is required to prevent premature death. Previous studies have suggested that ROS possibly influences and controls the life span of animals. For example, daf- 2 and isp- 1 mutants live longer because of their low levels of ROS [18]. Similarly, clk-1 mutants have low levels of accumulated byproducts from oxidative damage and extended life spans because of decreased ROS levels [19]. Mitochondria are major sources of ROS, and the mitochondria themselves can be damaged by ROS [30]. However, there is only indirect evidence linking genes encoded by mtDNA function with aging. This evidence is in the form of a series of mutations in nuclear genes that is sufficient to prove mtDNA involvement in the regulation of aging [21]. Therefore, we aimed to determine whether ROS and mtDNA influence the life span of lin-4 mutants.

Locomotion of $C$. elegans is controlled by a subset of its nervous system, and manipulations at the genetic or neuronal level allow an insight into the inner workings of this control [22]. Previous studies revealed that physiological levels of oxidative stress are associated with a balance between beneficial and harmful effects, and that normal levels of oxidative stress in C. elegans may be optimized for locomotor activity $[23,24]$. In the mature central nervous system, oxidative stress, calcium influx, and glutamate excitotoxicity can induce apoptosis. High ROS levels are capable of damaging cellular components, proteins, and nucleic acids and can even cause necrosis [25]. These facts imply that a loss-of-function mutation in lin-4 caused movement defects because of severe ROS accumulation. 


\section{Experimental Methods}

\subsection{Worms and Culture}

Wild-type C. elegans Bristol (N2) and lin-4 (e912) variant worms used in this study were obtained from the Caenorhabditis Genetics Center (Minnesota, USA). The lin-4 (e912) variant is a lin-4 null mutant. Worms were grown on NGM (Nematode Growth Medium) agar plated with E. coli OP50 at $20^{\circ} \mathrm{C}$.

\subsection{Nile Red Staining}

Nematodes were bred on media with $50 \mathrm{ng} / \mathrm{mL}$ Nile red (MP Biomedicals, CA, USA) and E. coli. After $72 \mathrm{~h}$ of culture, worms were collected, treated with $0.2 \%$ paraformaldehyde (PFA) solution, and observed under a fluorescence microscope (DMRXA; Leica) with N3 filter [27,28]. Images of Nile red staining were acquired using a Nikon camera under identical settings and exposure times to allow direct comparisons. The relative fluorescence of the whole nematode body was determined densitometrically using Image-Pro ${ }^{\circledR}$ Plus version 6.0, a commercially available software package (Media Cybernetics, Bethesda, MD, USA).

\subsection{Effect of Fatty Acid}

Worms were cultured on an NGM plate containing $1 \mathrm{mM}$ fatty acid (stearic acid, oleic acid, or linoleic acid; WAKO, JAPAN) and 0.1\% tergitol type NP-40 (Sigma, St. Louis, MO, USA) [12]. When the worms reached the young adult stage, they were subjected to Nile red analysis.

\subsection{Effect of Glucose}

Worms were cultured on an NGM plate with $5 \mathrm{mM}$ glucose, and were continually fed increasing doses of glucose until they reached the young adult stage [12,29]. Young adult C. elegans were subjected to Nile red analysis.

\subsection{Effect of Paraquat}

Worms were cultured on an NGM plate containing $2 \mathrm{mM}$ paraquat (Sigma) [30] and continued to grow into young adulthood with the dose. Young adult $C$. elegans were subjected to life span analysis.

\subsection{Measurement of Intracellular ROS in C. elegans}

Measurement of intracellular ROS in C. elegans was performed as previously described [31]. The amount of ROS was quantified using H2-DCF-DA (Sigma) as the molecular probe. H2-DCF-DA enters the cell, converts to H2-DCF, and is then rapidly oxidized by intracellular ROS to yield the fluorescent dye DCF. C. elegans worms were transferred to $2 \mathrm{~mL}$ of $\mathrm{M} 9$ buffer $\left(22 \mathrm{mmol} / \mathrm{L} \mathrm{KH}_{2} \mathrm{PO}_{4}\right.$, $22 \mathrm{mmol} / \mathrm{L} \mathrm{Na}_{2} \mathrm{HPO}_{4}, 85 \mathrm{mmol} / \mathrm{L} \mathrm{NaCl}$, and $1 \mathrm{mmol} / \mathrm{L} \mathrm{MgSO}_{4}$ ) that contained $10 \mu \mathrm{M} \mathrm{CM}-\mathrm{H} 2 \mathrm{DCFDA}$ and incubated for $30 \mathrm{~min}$ at $20{ }^{\circ} \mathrm{C}$. To determine the fluorescence of DCF, fixed nematode samples were analyzed using a fluorescence microscope (excitation at $488 \mathrm{~nm}$ and emission at $510 \mathrm{~nm}$ ). The 
relative fluorescence of the whole body was determined densitometrically using Image-Pro ${ }^{\circledR}$ Plus version 6.0, a commercially available software package (Media Cybernetics).

\subsection{Real-Time PCR of $m t D N A$}

The mtDNA copy number was determined by quantitative PCR assay as described previously [32]. The primer sets used to amplify mtDNA and chrDNA were described previously [33]. Sequences and TaqMan probes are shown in Table 1. DNA was prepared using a DNA extraction kit (Promega, Madison, WI, USA), and the samples were analyzed on a 7300 Real-Time PCR System (Applied Biosystems, Foster City, CA USA). All values were calculated using the absolute quantification method. The mtDNA copy number was normalized to the number of nuclear DNA copies.

Table 1. Sequences and TaqMan probes of mtDNA used in this study.

\begin{tabular}{lll}
\hline Symbol & Wormbase & Primers \\
\hline GDP2 & K10B3.8.1 & Probe: ACATAGTCGGCTCCAGAGGCTCC \\
(nuclear) & & Sense: CGCAGCACAAGATCAAGGTAG \\
& & Anti: AAGACTCCGGTGGACTCAAC \\
CYTB & Probe: GGGTCAGCTTCAATAAACATCTCTGC \\
(mitochondria) & Mitochondrial & Sense: GGTTATATTGCCACGGTGATTATG \\
& & Anti: AATATCACTCTGGAACAATATGAACTG \\
\hline
\end{tabular}

\subsection{Life Span and Locomotion Assay}

Life span assays were performed as described previously [34]. All life span analyses were conducted at $20{ }^{\circ} \mathrm{C}$, starting from the L4 stage to young adult-stage worms. Death was defined as failure to move after being prodded with a platinum wire. For analysis of locomotion, worms were transferred to an agar plate without a bacterial lawn. Locomotion rate was quantified by counting the number of body bends produced by the worms in 1 min. Body bends were counted by observing flexing in the middle of the worm body.

\subsection{Quantitative RT-PCR}

RNA extraction, purification, and reverse transcription were performed for each sample as described [35]. Quantitative RT-PCR using the universal TaqMan probe was performed, and the results were analyzed using a 7300 Real-Time PCR System (Applied Biosystems) under the following conditions: Samples were incubated at $95{ }^{\circ} \mathrm{C}$ for $10 \mathrm{~min}$ for initial denaturation, followed by 40 cycles of amplification that were performed at $95^{\circ} \mathrm{C}$ for $15 \mathrm{~s}$ and at $60{ }^{\circ} \mathrm{C}$ for $1 \mathrm{~min}$. All data were calculated using standard relative quantification $\Delta \Delta \mathrm{CT}$ methods [36], and actin was used as a control for normalization. All primers and probes for quantitative RT-PCR are listed in Table 2. 
Table 2. Sequences and target positions of the primers used for quantitative RT-PCR in this study.

\begin{tabular}{|c|c|c|}
\hline Symbol & Wormbase & Primers \\
\hline \multirow[t]{2}{*}{ Sbp-1 } & Y47D3B.7 & Probe: CTTCGCCGCCTTCTCCTTACTCCTCG \\
\hline & & Sense: CCACCACCTCATCACCACCAT \\
\hline \multirow[t]{2}{*}{ Oga-1 } & T20B5.3a & Anti: CCTTCCGCTGTCTCCTGAATCA \\
\hline & & Probe: CAGCCGAGTATTCACAGCCTATGGA \\
\hline \multirow[t]{5}{*}{ actin } & T04C12.5 & Sense: GAGCCTGTGCCTTCTGAGTTA \\
\hline & & Anti: CAGTGATAGTCTTTGATTTCTTATTTCCT \\
\hline & & Probe: CATCCTCCGTCTTGACTTGGCTGG \\
\hline & & Sense: CAATCTACGAAGGATATGCCCTC \\
\hline & & Anti: CTCAGCGGTGGTGGTGAA \\
\hline
\end{tabular}

\subsection{Statistical Analysis}

All data are shown as mean \pm SEM. Statistical analysis was performed using one-way ANOVA with the SPSS 12.0 statistical software package (SPSS Inc., USA). The level of significance was defined as $p<0.05$.

\section{Conclusion}

In this study, we found that a loss-of-function mutation in lin-4 in C. elegans led to a reduction in fat storage and decreased locomotion, and our findings also suggested that ROS may play an important role in the life span of lin-4 mutants.

\section{Acknowledgements}

This work was supported by grants from the National Natural Science Foundation of China (No. 30772364), the Natural Science Foundation of Jiangsu Province, China (No. BK2007230), the Foundation of Ministry of Education, China (No. 20070312001), and Nanjing Medical University (No. 07NMUZ024). We thank the Caenorhabditis Genetics Center (University of Minnesota) for providing strains.

\section{References}

1. Bartel, D.P. MicroRNAs: genomics, biogenesis, mechanism, and function. Cell 2004, 116, 281-297.

2. Ambros, V. The functions of animal microRNAs. Nature 2004, 431, 350-355.

3. Wienholds, E.; Plasterk, R.H. MicroRNA function in animal development. FEBS Lett. 2005, 579, 5911-5922.

4. Heneghan, H.M.; Miller, N.; Kerin, M.J. Role of microRNAs in obesity and the metabolic syndrome. Obes. Rev. 2010, 11, 354-361.

5. Vella, M.C.; Slack, F.J.C. Elegans microRNAs. Worm Book 2005, 21, 1-9. 
6. Olsen, P.H.; Ambros, V. The lin-4 regulatory RNA controls developmental timing in Caenorhabditis elegans by blocking LIN-14 protein synthesis after the initiation of translation. Dev. Biol. 1999, 216, 671-680.

7. Boehm, M.; Slack, F. A developmental timing microRNA and its target regulate life span in C. elegans. Science 2005, 310, 1954-1957.

8. Singhal, V.; Schwenk, W.F; Kumar, S. Evaluation and management of childhood and adolescent obesity. Mayo Clin. Proc. 2007, 82, 1258-1264.

9. Speakman, J.; Hambly, C.; Mitchell, S.; Krol, E. The contribution of animal models to the study of obesity. Lab. Anim. 2008, 42, 413-432.

10. McKay, R.M.; McKay, J.P.; Avery, L.; Graff, J.M. C elegans: A model for exploring the genetics of fat storage. Dev. Cell 2003, 4, 131-142.

11. Brooks, K.K.; Liang, B.; Watts. J.L. The influence of bacterial diet on fat storage in C. elegans. PLoS One 2009, 4, e7545.

12. Nomura, T.; Horikawa, M.; Shimamura, S.; Hashimoto, T.; Sakamoto, K. Fat accumulation in Caenorhabditis elegans is mediated by SREBP homolog SBP-1. Genes Nutr. 2009, 5, 17-27.

13. Forsythe, M.E.; Love, D.C.; Lazarus, B.D.; Kim, E.J.; Prinz, W.A.; Ashwell, G.; Krause M.W.; Hanover J.A. Caenorhabditis elegans ortholog of a diabetes susceptibility locus: oga-1 (O-GlcNAcase) knockout impacts O-GlcNAc cycling, metabolism, and dauer. Proc. Natl. Acad. Sci. USA 2006, 103, 11952-11957.

14. Eberlé, D.; Hegarty, B.; Bossard, P.; Ferré, P.; Foufelle, F. SREBP transcription factors: Master regulators of lipid homeostasis. Biochimie 2004, 86, 839-848.

15. Yang, F.; Vought, B.W.; Satterlee, J.S.; Walker, A.K.; Jim Sun, Z.Y.; Watts, J.L.; DeBeaumont, R.; Saito, R.M.; Hyberts, S.G.; Yang, S.; Macol, C.; lyer, L.; Tjian, R.; van den Heuvel, S.; Hart, A.C.; Wagner, G.; Naar, A.M. An ARC/Mediator subunit required for SREBP control of cholesterol and lipid homeostasis. Nature 2006, 442, 700-704.

16. Wells, L.; Whelan, S.A.; Hart, G.W. O-GlcNAc: A regulatory post-translational modification. Biochem. Biophys. Res. Commun. 2003, 302, 435-441.

17. González-Polo, R.A.; Rodríguez-Martín, A.; Morán, J.M.; Niso, M.; Soler, G.; Fuentes, J.M. Paraquat-induced apoptotic cell death in cerebellar granule cells. Brain Res. 2004, 1011, 170-176.

18. Hekimi, S.; Guarente, L. Genetics and the specificity of the aging process. Science 2003, 299, 1351-1354.

19. Braeckman, B.P.; Houthoofd, K.; Brys, K.; Lenaerts, I.; de Vreese, A.; van Eygen, S.; Raes, H.; Vanfleteren, J.R. No reduction of energy metabolism in Clk mutants. Mech. Ageing Dev. 2003, $123,1447-1456$.

20. Ide, T.; Tsutsui, H.; Hayashidani, S.; Kang, D.; Suematsu, N.; Nakamura, K.; Utsumi, H.; Hamasaki, N.; Takeshita, A. Mitochondrial DNA damage and dysfunction associated with oxidative stress in failing hearts after myocardial infarction. Circ. Res. 2001, 88, 529-535.

21. Beckman, K.B.; Ames, B.N. The free radical theory of aging matures. Physiol. Rev. 1998, 78, 547-581.

22. Boyle, J.H.; Cohen, N. Caenorhabditis elegans body wall muscles are simple actuators. Biosyststems 2008, 94, 170-181. 
23. Finkel, T.; Holbrook, N.J. Oxidants, oxidative stress and the biology of aging. Nature 2000, 408, 239-247.

24. Murakami, S.; Murakami, H. The effects of aging and oxidative stress on learning behavior in C. elegans. Neurobiol. Aging 2005, 26, 899-905.

25. Kam, P.C.A.; Ferch, N.I. Apoptosis: Mechanisms and clinical implications. Anaesthesia 2000, 55, 1081-1093.

26. Libina, N.; Berman, J.R.; Kenyon, C. Tissue-specific activities of C. elegans DAF-16 in the regulation of lifespan. Cell 2006, 115, 489-502.

27. Hashimoto, T.; Horikawa, M.; Nomura, T.; Sakamoto, K. Nicotinamide adenine dinucleotide extends the lifespan of Caenorhabditis elegans mediated by sir-2.1 and daf-16. Biogerontology 2010, 11, 31-43.

28. Horikawa, M.; Nomura, T.; Hashimoto, T.; Sakamoto, K. Elongation and desaturation of fatty acids are critical in growth, lipid metabolism, and ontogeny of Caenorhabditis elegans. J. Biochem. 2008, 144, 149-158.

29. Lee, S.J.; Murphy, C.T.; Kenyon, C. Glucose shortens the life span of C. elegans by downregulating DAF-16/FOXO activity and aquaporin gene expression. Cell Metab. 2009, 10, 379-391.

30. Keaney, M.; Matthijssens, F.; Sharpe, M.; Vanfleteren, J.; Gems, D. Superoxide dismutase mimetics elevate superoxide dismutase activity in vivo but do not retard aging in the nematode Caenorhabditis elegans. Free Radic. Biol. Med. 2004, 37, 239-250.

31. Kampkötter, A.; Nkwonkam, C.G.; Zurawski, R.F.; Timpel, C.; Chovolou, Y.; Watjen, W.; Kahl, R. Investigations of protective effects of the flavonoids quercetin and rutin on stress resistance in the model organism Caenorhabditis elegans. Toxicology 2007, 234, 113-123.

32. Miller, K.G.; Alfonso, A.; Nguyen, M.; Crowell, J.A.; Johnson, C.D.; Rand, J.B. A genetic selection for Caenorhabditis elegans synaptic transmission mutants. Proc. Natl. Acad. Sci. USA 1996, 93, 12593-12598.

33. Ye, K.; Ji, C.B.; Lu, X.W.; Ni, Y.H.; Gao, C.L.; Chen, X.H.; Zhao, Y.P.; Gu, G.X.; Guo, X.R. Resveratrol attenuates radiation damage in Caenorhabditis elegans by preventing oxidative stress. J. Radiat. Res. 2010, 51, 473-479.

34. Dillin, A.; Crawford, D.K.; Kenyon, C. Timing requirements for insulin/IGF-1 signaling in C. elegans. Science 2002, 298, 830-834.

35. Taubert, S.; van Gilst, M.R.; Hansen, M.; Yamamoto, K.R. A Mediator subunit, MDT-15, integrates regulation of fatty acid metabolism by NHR-49-dependent and independent pathways in C. elegans. Genes Dev. 2006, 20, 1137-1149.

36. Livak, K.J.; Schmittgen, T.D. Analysis of relative gene expression data using real-time quantitative PCR and the 2(-Delta Delta C(T)) method. Methods 2001, 25, 402-408.

(C) 2010 by the authors; licensee MDPI, Basel, Switzerland. This article is an open access article distributed under the terms and conditions of the Creative Commons Attribution license (http://creativecommons.org/licenses/by/3.0/). 